\title{
The Trigonometric Rosen-Morse Potential in the Supersymmetric Quantum Mechanics and its Exact Solutions
}

\author{
C. B. Compean and M. Kirchbach \\ Instituto de Física, \\ Universidad Autónoma de San Luis Potosí, \\ Av. Manuel Nava 6, San Luis Potosí, \\ S.L.P. 78290, México
}

(Dated: February 1, 2008)

\begin{abstract}
The analytic solutions of the one-dimensional Schrödinger equation for the trigonometric RosenMorse potential reported in the literature rely upon the Jacobi polynomials with complex indices and complex arguments. We first draw attention to the fact that the complex Jacobi polynomials have non-trivial orthogonality properties which make them uncomfortable for physics applications. Instead we here solve above equation in terms of real orthogonal polynomials. The new solutions are used in the construction of the quantum-mechanical superpotential.
\end{abstract}

PACS numbers: 02.30.Gp, 03.65.Ge, 12.60.Jv 


\section{INTRODUCTION}

Supersymmetric quantum mechanics was originally proposed by Witten [1] as a simple learning ground for the basic concepts of supersymmetric quantum field theories but soon after it evolved to a research field on its own rights. Supersymmetric quantum mechanics starts with the factorization of one-dimensional Hamiltonians,

$$
H(y)=-\frac{\hbar^{2}}{2 m} \frac{d^{2}}{d y^{2}}+V(y)
$$

according to $H(y)=A^{+}(y) A^{-}(y)+\epsilon$ with $A^{ \pm}(y)=\left( \pm \frac{\hbar}{\sqrt{2 m}} \frac{d}{d y}+U(y)\right)$ where $U(y)$ is the superpotential. Next it proves that if $\psi_{n}(y)$ is an exact solution of the $H(y)$ eigenvalue problem, $H(y) \psi_{n}(y)=E_{n} \psi_{n}(y)$, then $A(y) \psi_{n}(y)$ is an eigenfunction to $\widetilde{H}(y)=A^{-}(y) A^{+}(y)+\epsilon$ corresponding to same eigenvalue. In other words, knowing the superpotential allows to generate the $\widetilde{H}(y)$ spectrum from the spectrum of $H(y)$ and vise versa. Moreover, in case of zero ground state (gst) energy, knowing $U(y)$ allows to solve $A^{-}(y) \psi_{\text {gst }}(y)=0$ and obtain the ground state wave function. In other words, knowing the ground state wave function allows to recover the superpotential as

$$
U(y)=-\frac{\hbar}{\sqrt{2 m}} \frac{d}{d y} \ln \psi_{\mathrm{gst}}(y)
$$

At that stage one uses the isospectral pair $H(y)$, and $\widetilde{H}(y)$ in the construction of the superHamiltonian $\mathbf{H}(\mathrm{y})$ as $\mathbf{H}(\mathrm{y})=\operatorname{diag}(H(y), \widetilde{H}(y))$ and upon introducing "charges" as

$$
Q(y)=\left(\begin{array}{cc}
0 & 0 \\
A^{-}(y) & 0
\end{array}\right), \quad Q^{\dagger}(y)=\left(\begin{array}{cc}
0 & A^{+}(y) \\
0 & 0
\end{array}\right)
$$

proves them to satisfy the following algebra:

$$
\begin{gathered}
\left\{Q(y), Q^{\dagger}(y)\right\}=\mathbf{H}(y), \quad\{Q(y), Q(y)\}=\left\{Q^{\dagger}(y), Q^{\dagger}(y)\right\}=0 \\
{[Q(y), \mathbf{H}(y)]=\left[Q^{\dagger}(y), \mathbf{H}(y)\right]=0}
\end{gathered}
$$

The relationship to the field-theoretic SUSY is then established through the observation that in case $A^{-}(y) \psi_{\text {gst }}(y) \neq 0$ then the charges do not annihilate the respecive vacua, $Q(y) \psi_{\text {gst }}(y) \neq 0$, and $Q^{\dagger}(y) \widetilde{\psi}_{\text {gst }}(y) \neq 0$, and SUSY is spontaneously broken. On the contrary, when $A^{-}(y) \psi_{\text {gst }}(y)=0$, then the charges annihilate the ground states which is equivalent to the absence of charge condensates there, and thereby to SUSY realization 
in the manifest (multiplet) Wigner-Weyl mode. In this manner supersymmetric quantum mechanics relates to SUSY in field theory where the róle of $Q(y)$ and $Q^{\dagger}(y)$ is taken by boson-fermion (and vise versa) ladder operators.

Above considerations clearly reveal importance of knowing the exact solutions of the quantum mechanics Hamiltonians. These solutions are furthermore important in the construction of higher dimensional charge algebras with more but two Hamiltonians (hierarchy of Hamiltonians) 2]. The supersymmetric quantum mechanics manages a family of exactly soluble

potentials [3], one of them being the trigonometric Rosen-Morse potential (tRMP). As long as this potential is obtained from the Eckart potential [4] by complexification of the argument and one of the constants, also its solutions have been concluded from those of the Eckart potential by same procedure. In so doing, one ends up with Jacobi polynomials with complex indices and complex arguments. However, such complex polynomials are not comfortable for physical applications basically in view of their non-trivial orthogonality properties [5, 6].

We here make the case that the trigonometric Rosen-Morse potential is exactly soluble in terms of a family of real orthogonal polynomials and present the solutions.

The paper is organized as follows. In the next Section we present the tRMP derivation form the Eckart potential and draw attention to the non-trivial orthogonality properties of the Jacobi polynomials with complex parameters and arguments. In Section III we solve analytically the one-dimensional Schrödinger equation with the trigonometric Rosen-Morse potential and present the solutions. In Section IV we employ the exact ground state wave function in the construction of the tRMP superpotential. The paper closes with brief concluding remarks.

\section{THE TRIGONOMETRIC ROSEN-MORSE POTENTIAL AS COMPLEXIFIED ECKART POTENTIAL.}

Before proceeding further we first introduce a properly chosen length scale $d$ and change variables in the one-dimensional Schrödinger equation (11) to dimensionless ones according to

$$
z=\frac{y}{d}, \quad v(z)=V(d z) /\left(\hbar^{2} / 2 m d^{2}\right), \quad \epsilon_{n}=E_{n} /\left(\hbar^{2} / 2 m d^{2}\right)
$$


Next we employ the Eckart potential [4],

$$
v(z)=-2 b \operatorname{coth} z+a(a-1) \operatorname{csch}^{2} z,
$$

where $b>a^{2}$. The exact solutions to the Eckart potential read:

$$
\begin{aligned}
\psi_{n}(x)= & c_{n}(x-1)^{\left(\beta_{n}-n-a\right) / 2}(x+1)^{-\left(\beta_{n}+n+a\right) / 2} P_{n}^{\beta_{n}-n-a,-\left(\beta_{n}+n+a\right)}(x), \\
x=\operatorname{coth} z, \quad & \beta_{n}=\frac{b}{n+a} .
\end{aligned}
$$

Here, $P_{n}^{\left(\beta_{n}-n-a,-\left(\beta_{n}+n+a\right)\right)}(x)$ are the well known Jacobi polynomials [7], $\left[\right.$ 8] with $n \leq\left(b^{1 / 2}-a\right)$, and $c_{n}$ is a normalization constant. Equation (77) equivalently rewrites to

$$
\psi_{n}(x)=c_{n}\left(x^{2}-1\right)^{-(n+a) / 2} \mathrm{e}^{-\beta_{n} \operatorname{arccoth} x} P_{n}^{\left(\beta_{n}-n-a,-\left(\beta_{n}+n+a\right)\right)}(x) .
$$

The corresponding energy value spectrum is determined by

$$
\epsilon_{n}=-(n+a)^{2}-\frac{b^{2}}{(n+a)^{2}} .
$$

Form and energy spectrum of the Eckart potential are illustrated by Fig. I. Let's now

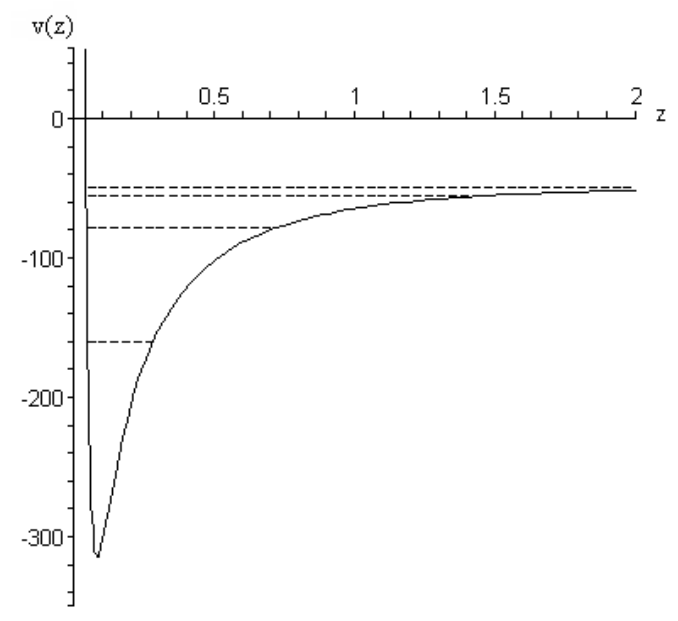

Fig. I. Eckart potential. The solid curve represents the potential while the dashed lines are the energy levels. Notice that the argument of this potential is unbound from above, i.e. $0<z<\infty$ and the number of bound states limited. The parameters of the displayed potential take the

$$
\text { values } a=-1, b=50 \text {. }
$$

complexify the argument of the Eckart potential and one of its constants according to

$$
z \rightarrow-i z, \quad \text { or, equivalently, } \quad x \longrightarrow i x ; \quad b \rightarrow i b .
$$


Substitution of Eq. (10) into (6) results in

$$
v(z)=-2 b \cot z+a(a-1) \csc ^{2} z,
$$

and thereby in the trigonometric Rosen-Morse potential 3] shown on Fig. [I]

In the literature [3] the solution of the Schrödinger equation with the trigonometric Rosen-

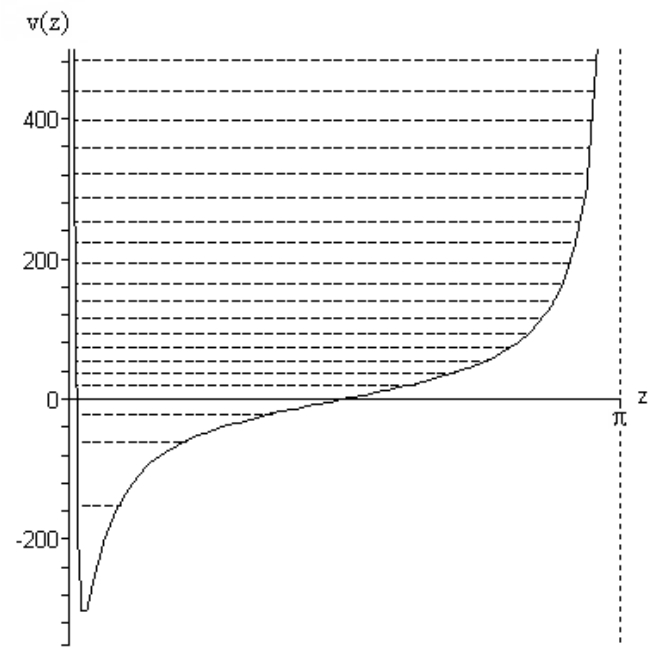

Fig. II. The trigonometric Rosen-Morse potential. The solid line represents the potential, while the dashed lines are the energy levels. Notice that the argument of this potential is bound between $0<z<\pi$ and the number of states is unlimited. The potential parameters take the

$$
\text { values } a=1, b=50 \text {. }
$$

Morse potential is concluded from Eq. (8) through complexification of $b$ and $x$ leading to

$$
\psi_{n}(i x)=c_{n}\left((i x)^{2}-1\right)^{-(n+a) / 2} \mathrm{e}^{-\beta_{n} \operatorname{arccoth} i x} P_{n}^{\left(\left(i \beta_{n}-n-a\right),-\left(i \beta_{n}+n+a\right)\right)}(i x) .
$$

In other words, these solutions need the Jacobi polynomials with complex indices and complex arguments. Unfortunately, the complex Jacobi polynomials are not very well suited for physics applications. Suffices to write down these polynomials explicitly,

$$
\begin{aligned}
& P_{n}^{(A, B)}(i x)=\frac{2^{n}(A+1)_{n}}{(n+A+B+1)_{n}} \quad{ }_{2} F_{1}\left(-n, n+A+B+1 ; A+1 \mid \frac{1-i x}{2}\right), \\
& { }_{2} F_{1}\left(-n, n+A+B+1 ; A+1 \mid \frac{1-i x}{2}\right)=\frac{\Gamma(A+1)}{\Gamma(n+A+B+1) \Gamma(-(n+B))} \\
& \int_{0}^{1} t^{n+A+B}(1-t)^{-(n+B+1)}\left(1-t \frac{1-i x}{2}\right)^{n} d t \\
& A=i \beta_{n}-n-a, \quad B=-\left(i \beta_{n}+n+a\right),
\end{aligned}
$$


where ${ }_{2} F_{1}(a, b ; c \mid x)$ is the well known hypergeometric function, and $(\ldots)_{n}$ is the Pochhammer symbol of the expression in the parentheses, in order to become aware of the calculational difficulties to be expected. It is obvious that one has to worry about the interplay between indices and integration contours, a subject studied in [5, 6]. There the authors claim dependence of the orthogonality properties on the indices and the integration contours. In order to avoid all those difficulties we here search for real solutions of the one-dimensional Schrödinger equation with the trigonometric Rosen-Morse potential.

\section{THE ONE-DIMENSIONAL SCHRÖDINGER EQUATION FOR THE TRIGONOMETRIC ROSEN-MORSE POTENTIAL.}

\section{A. The Sturm-Liouville method and the Rodrigues formula.}

For the sake of self-sufficiency of the representation we here review in brief the basics of the Sturm-Liuoville technique for solving second order differential equations.

The method of Sturm-Liouville [9] applies to differential equations of the type

$$
\frac{d}{d x}\left(p(x) \frac{d y}{d x}\right)+q(x) y=\lambda w(x) y,
$$

and searches for the $\lambda$ values that allow for a solution. The solution of Eq. (14), where $w(x)$ stands for the "weight", or, "density" function, are eigenfunctions of a Hermitian differential operator on the space of functions defined by the initially conditions. Some special cases of the Sturm-Liouville type of differential equations allow for solutions by means of the Rodrigues formula [7]. To be specific, for $q(x)=0$, and $p(x)=w(x) s(x)$, where $s(x)$ at most a second order polynomial, the solution of Eq. (14) is given by a family of orthogonal polynomials. The classical polynomials of Hermite, Laguerre, Legendre and Jacobi are prominent examples for that.

In order to create the orthogonal polynomial solutions one considers the function [7]

$$
C_{m}(x)=\frac{1}{w(x)} \frac{d^{m}}{d x^{m}}\left(w(x) s(x)^{m}\right)
$$

with $C_{1}(x), w(x)$, and $s(x)$ satisfying the following conditions:

1. $C_{1}(x)$ is a polynomial of first order, 
2. $s(x)$ is a polynomial of at most second order and real roots,

3. $w(x)$ is real, positive and integrable within a given interval $[a, b]$, and satisfies the boundary conditions

$$
w(a) s(a)=w(b) s(b)=0
$$

Above three conditions seem quite restrictive indeed but nonetheless they allow for the construction of all the classical orthogonal polynomials reported in the standard textbooks [7], 8], [9]. The table shows all the ingredients of the Rodrigues formula required for the construction of the respective orthogonal polynomials.

TABLE I: Special functions and their characteristics.

\begin{tabular}{||l|c|c|c|c|l||}
\hline \hline Name & Symbol & $w(x)$ & $s(x)$ & Interval & Conditions \\
\hline \hline Hermite & $H_{m}(x)$ & $\mathrm{e}^{-x^{2}}$ & 1 & $(-\infty, \infty)$ & \\
\hline Laguerre & $L_{m}^{\nu}(x)$ & $x^{\nu} \mathrm{e}^{-x}$ & $x$ & {$[0, \infty)$} & $(\nu>-1)$ \\
\hline Jacobi & $P_{m}^{(\nu, \mu)}(x)$ & $(1-x)^{\nu}(1+x)^{\mu}$ & $\left(1-x^{2}\right)$ & {$[-1,1]$} & $(\nu, \mu>-1)$ \\
\hline Gegenbauer & $C_{m}^{\lambda}(x)$ & $\left(1-x^{2}\right)^{\lambda-1 / 2}$ & $\left(1-x^{2}\right)$ & {$[-1,1]$} & $(\lambda>-1 / 2)$ \\
\hline Legendre & $P_{m}(x)$ & 1 & $\left(1-x^{2}\right)$ & {$[-1,1]$} & \\
\hline Chebyshev, Type I & $T_{m}(x)$ & $\left(1-x^{2}\right)^{-1 / 2}$ & $\left(1-x^{2}\right)$ & {$[-1,1]$} & \\
\hline Chebyshev, Type II & $U_{m}(x)$ & $\left(1-x^{2}\right)^{1 / 2}$ & $\left(1-x^{2}\right)$ & {$[-1,1]$} & \\
\hline \hline This work & $C_{m+1}^{(a, b)}(x)$ & $\left(1+x^{2}\right)^{-\mu} \mathrm{e}^{-2 \frac{b}{\mu} \cot ^{-1} x}$ & $\left(1+x^{2}\right)$ & $(-\infty, \infty)$ & $(\mu=m+1+a)$ \\
\hline \hline
\end{tabular}

If in addition one demands an orthonormalized set of polynomials, one has to introduce into Eq. (15) an additional constant, here denoted by $K_{m}$, according to

$$
C_{m}(x)=\frac{1}{K_{m} w(x)} \frac{d^{m}}{d x^{m}}\left(w(x) s(x)^{m}\right) .
$$

In terms of $C_{m}(x)$, the Sturm-Liouville equation takes the form

$$
\frac{d}{d x}\left(w(x) s(x) \frac{d C_{m}(x)}{d x}\right)=-\lambda_{m} w(x) C_{m}(x)
$$

or, equivalently,

$$
s(x) \frac{d^{2} C_{m}(x)}{d x^{2}}+\frac{1}{w(x)}\left(\frac{d s(x) w(x)}{d x}\right) \frac{d C_{m}(x)}{d x}+\lambda_{m} C_{m}(x)=0,
$$


where

$$
\lambda_{m}=-m\left(K_{1} \frac{d C_{1}(x)}{d x}+\frac{1}{2}(m-1) \frac{d^{2} s(x)}{d x^{2}}\right) .
$$

Notice that

$$
C_{1}(x)=\frac{1}{K_{1} w(x)}\left(\frac{d s(x) w(x)}{d x}\right) .
$$

All classical polynomials can be obtained through above procedure and vise versa. The polynomials that can be obtained from the Rodrigues formula and which satisfy the three conditions mentioned above are necessarily the classical orthogonal polynomials, a result due to Ref. [10].

\section{B. Solving Schrödinger's equation for the trigonometric Rosen-Morse potential.}

In this Section we present the solution of the one-dimensional Schrödinger equation for the trigonometric Rosen-Morse potential as obtained in Ref. 11] and without any reliance

on the complex Jacobi polynomials. For this purpose we first have to reshape Schrödinger's equation

$$
\frac{d^{2} R(z)}{d z^{2}}+\left(2 b \cot z-a(a+1) \csc ^{2} z+\epsilon\right) R(z)=0
$$

to the Sturm-Liouville form in Eq. (19). To do so we first change variables to

$$
R(z)=\mathrm{e}^{-\alpha z / 2} F(z),
$$

with $\alpha$ being a constant and then substitute in Eq. (22). After some simple algebraic manipulations one arrives at

$$
\frac{d^{2} F(z)}{d z^{2}}-\alpha \frac{d F(z)}{d z}+\left(2 b \cot z-a(a+1) \csc ^{2} z+\left(\left(\frac{\alpha}{2}\right)^{2}+\epsilon\right)\right) F(z)=0 .
$$

Changing once more variables to $x=\cot z$ in which case $F(z)$ becomes a function of $x$ denoted by $f(x)$, i.e.

$$
F(z) \rightarrow f(x), \quad x=\cot z,
$$

Eq. (24) takes the form

$$
\begin{aligned}
\left(2 b x-a(a+1)\left(1+x^{2}\right)+\left(\left(\frac{\alpha}{2}\right)^{2}+\epsilon\right)\right) f(x) & +\left(1+x^{2}\right)^{2} \frac{d^{2} f(x)}{d x^{2}} \\
& +2\left(1+x^{2}\right)\left(\frac{\alpha}{2}+x\right) \frac{d f(x)}{d x}=0
\end{aligned}
$$


Finally, upon substituting the factorization ansatz

$$
f(x)=\left(1+x^{2}\right)^{-(1-\beta) / 2} C(x),
$$

into Eq. (26) and a subsequent division by $\left(1+x^{2}\right)^{(1+\beta) / 2}$ one finds as intermediate result the equation

$$
\begin{array}{r}
\left((-\beta(1-\beta)-a(a+1))+\frac{(-\alpha(1-\beta)+2 b) x+\left(\left(\frac{\alpha}{2}\right)^{2}-(1-b)^{2}+\epsilon\right)}{\left(1+x^{2}\right)}\right) C(x) \\
+\left(1+x^{2}\right) \frac{d^{2} C(x)}{d x^{2}}+2\left(\frac{\alpha}{2}+\beta x\right) \frac{d C(x)}{d x}=0 .
\end{array}
$$

If Eq. (28) is to coincide in form with Eq. (19), following conditions have to be fulfilled:

$$
\begin{aligned}
-\alpha(1-\beta)+2 b & =0 \\
\left(\frac{\alpha}{2}\right)^{2}-(1-\beta)^{2}+\epsilon & =0 .
\end{aligned}
$$

Substitution of Eqs. (29), (30) into Eq. (28) amounts to

$$
\left(1+x^{2}\right) \frac{d^{2} C(x)}{d x^{2}}+2\left(\frac{\alpha}{2}+\beta x\right) \frac{d C(x)}{d x}+(-\beta(1-\beta)-a(a+1)) C(x)=0 .
$$

Equation (31) relates to Eqs. (19)-(21) via

$$
-\beta(1-\beta)-a(a+1)=-m(2 \beta+m-1) .
$$

In now determining $\beta$ from Eq. (32), substituting it into Eqs. (29), (301), and shifting $m$ to $m \rightarrow n-1$, the following $n$ dependent constants are found:

$$
\begin{aligned}
\beta_{n}=-(n+a)+1, & \alpha_{n}=\frac{2 b}{n+a} \\
\epsilon_{n}= & (n+a)^{2}-\frac{b^{2}}{(n+a)^{2}},
\end{aligned}
$$

with $n \geq 1$.

In this way one encounters $w(x)$ and $s(x)$ as

$$
\begin{aligned}
w_{n}(x) & =\left(1+x^{2}\right)^{-(n+a)} \mathrm{e}^{-\alpha_{n} \operatorname{arccot} x}, \\
s(x) & =1+x^{2} .
\end{aligned}
$$


The polynomials which resolve the tRMP Schrödinger equation are now obtained in exploiting the Rodrigues formula (17) when rewritten in terms of $n$ as

$$
C_{n}^{(a, b)}(x)=\frac{1}{K_{n} w(x)} \frac{d^{n-1}}{d x^{n-1}}\left(w_{n}(x) s(x)^{n-1}\right) .
$$

The lowest $C_{n}^{(a, b)}(x)$ polynomials obtained in this fashion read

$$
\begin{aligned}
C_{1}^{(a, b)}(x)= & \frac{1}{K_{1}} \\
C_{2}^{(a, b)}(x)= & \frac{2}{K_{2}}\left(-(1+a) x+\frac{b}{2+a}\right) \\
C_{3}^{(a, b)}(x)= & \frac{2}{K_{3}}\left((1+a)(2 a+3) x^{2}-2(2 a+3) \frac{b}{3+a} x+\left(\frac{2 b^{2}}{(3+a)^{2}}-(1+a)\right)\right) \\
C_{4}^{(a, b)}(x)= & \frac{4}{K_{4}}\left(-(1+a)(2 a+3)(2+a) x^{3}+3(a+2)(2 a+3) \frac{b}{(4+a)} x^{2}\right. \\
& \left.-3(2+a)\left(2 \frac{b^{2}}{(4+a)^{2}}-(1+a)\right) x+\left(\frac{2 b^{3}}{(4+a)^{3}}-(3 a+4) \frac{b}{4+a}\right)\right) \\
C_{5}^{(a, b)}(x)= & \frac{4}{K_{5}}\left((1+a)(2 a+3)(2+a)(2 a+5) x^{4}-4(2 a+3)(2+a)(2 a+5) \frac{b}{(5+a)} x^{3}\right. \\
& +6(2+a)(2 a+5)\left(\frac{2 b^{2}}{(5+a)^{2}}-(1+a)\right) x^{2} \\
& -4(2 a+5)\left(\frac{2 b^{3}}{(5+a)^{3}}-(3 a+4) \frac{b}{5+a}\right) x \\
& \left.+\left(\frac{4 b^{4}}{(5+a)^{4}}-\frac{4 b^{2}}{(5+a)^{2}}(3 a+5)+3(2+a)(1+a)\right)\right)
\end{aligned}
$$

where $x=\cot z$.

Above polynomials solve exactly Eq. (31) which can be immediately cross-checked by backsubstituting Eqs. (42) into Eq. (31). Employing symbolic mathematical programs is quite useful in that regard.

Notice that the solution was found under less rigid requirements but the ones listed immediately after Eq. (15) above. Indeed,

- the roots of our $s(x)$ function are imaginary (this is the only place where the complexification of the Eckart potential seems to have left footprints), - Equation (16) turned out to be a rule that allows to be broken.

In that sense, the orthogonal functions found here must belong to a different class of orthogonal functions. 
Their orthogonality is obtained as

$$
\int_{-\infty}^{\infty} \frac{d x}{s(x)}\left(w_{n}(x)\right)^{1 / 2} C_{n}^{(a, b)}\left(w_{n^{\prime}}(x)\right)^{1 / 2} C_{n^{\prime}}^{(a, b)}=\delta_{n n^{\prime}} .
$$

Equation (43) shows convincingly that the new solutions have well defined orthogonality properties on the real axes, which qualifies them as comfortable wave functions in quantum mechanics applications.

The orthogonality condition for the wave functions $R_{n}(z)$ reads

$$
\int_{0}^{\pi} d z R_{n}(z)\left(R_{n^{\prime}}(z)\right)^{*}=\delta_{n n^{\prime}}
$$

A version interesting for physical application (see concluding Section) is the one with $a=0$,

$$
v(z)=-2 b \cot z
$$

in which case the normalization constant is calculated as

$$
K_{n}=\left(\frac{(n !)^{2} n^{3}\left(1-\mathrm{e}^{-2 \pi b / n}\right)}{4 b\left(b^{2}+n^{4}\right)}\right)^{1 / 2} .
$$

The associated energy spectrum is given then by

$$
\epsilon_{n}=n^{2}-\frac{b^{2}}{n^{2}}
$$

Correspondingly, the wave functions for this case simplify and are found as

$$
\begin{aligned}
R_{1}(z) & =\mathrm{e}^{-b z} \sin z C_{1}^{(0, b)}(\cot z), \\
R_{2}(z) & =\mathrm{e}^{-b z / 2} \sin ^{2} z C_{2}^{(0, b)}(\cot z), \\
\cdots & \\
R_{n}(z) & =\mathrm{e}^{-b z / n} \sin ^{n} z C_{n}^{(0, b)}(\cot z) .
\end{aligned}
$$

Wave functions for the first two (unnormalized) levels with $a \neq 0$ are displayed in Figs. III.

\section{THE TRIGONOMETRIC ROSEN-MORSE SUPERPOTENTIAL.}

In this Section we derive the trigonometric Rosen-Morse superpotential from the exact ground state solution which for $a \neq 0$ has been calculated as

$$
R_{1}(z) \propto \mathrm{e}^{-b z /(a+1)} \sin ^{a+1} z
$$



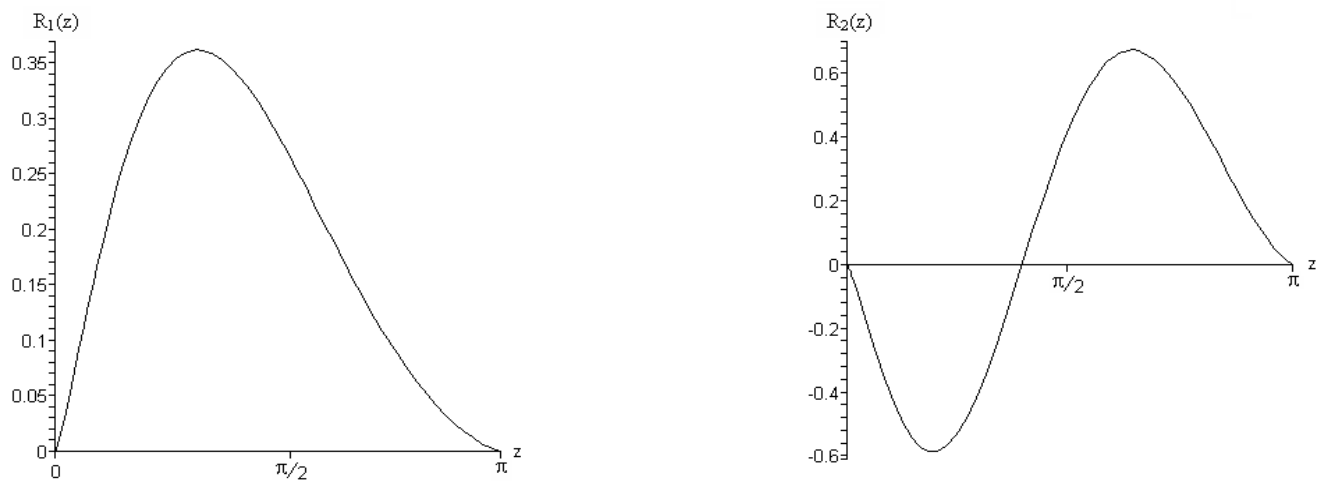

Fig. III. Wave functions for the first two levels in the trigonometric Rosen-Morse potential for $a=0.25$ and $b=1$.

Substitution of the latter equation (49) into Eq. (2) amounts to the following superpotential,

$$
U(z)=-\frac{b}{a+1}+(a+1) \cot z,
$$

shown in Fig IV. Correspondingly, the $A^{ \pm}(z)$ operators are obtained as

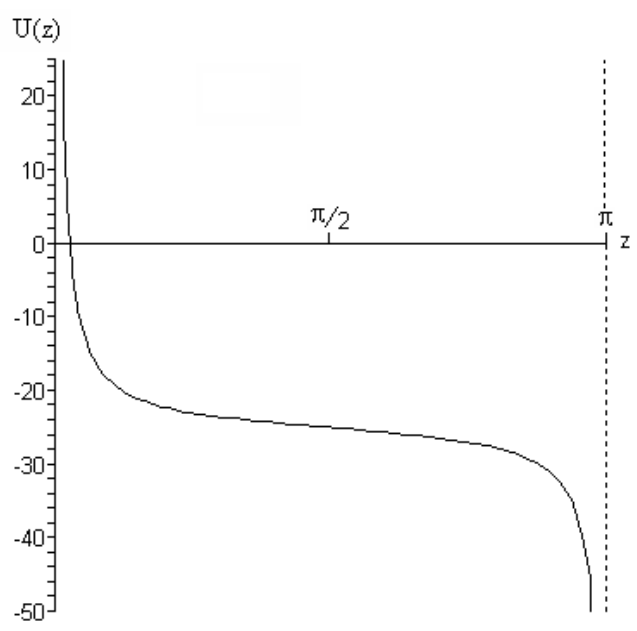

Fig. IV. The trigonometric Rosen-Morse superpotential. The values of the displayed superpotential parameters are same as in Fig. III

$$
A^{ \pm}(z)= \pm \frac{d}{d z}+(a+1) \cot z-\frac{b}{a+1} .
$$

The corresponding Hamiltonian is then obtained identical to Eq. (11), as should be. The supersymmetric companion of $H(z)$, which is $\widetilde{H}(z)$, becomes

$$
\widetilde{H}(z)=-\frac{d^{2}}{d z^{2}}-2 b \cot z+(a+1)(a+2) \csc ^{2} z,
$$


and one finds the supersymmetric companions to the solutions as

$$
\left.\widetilde{R}_{n}(z)\right|_{(a, b)}=\left.R_{n-1}(z)\right|_{(a+1, b)},
$$

where $n>1$, and $\left.R_{n-1}(z)\right|_{(a+1, b)}$ are among the exact solutions of the trigonometric RosenMorse potential.

As a further possible application of the solutions found here we wish to mention the construction of the so called hierarchy of Hamiltonians [2], where one needs to have at ones disposal exact orthonormalized functions for all levels because in this case one can pick up any energy level, $\epsilon_{n}$, and its wave function $R_{n}(z)$. Obviously, Eq. (48) fully qualify for that purpose.

\section{CONCLUDING REMARKS.}

To recapitulate, the knowledge on the real exact solutions of the one-dimensional Schrödinger equation for the trigonometric Rosen-Morse potential obtained in this work allows for a straightforward construction of all the necessary ingredients of the supersymmetric quantum mechanics. Compared to the text-book complex solutions, the exact real solutions reported here have the advantage to significantly simplify the calculations.

In addition, the trigonometric Rosen-Morse potential is more but just one of the few exactly soluble quantum mechanical potentials. It joins as a new member the smaller and important group of potentials which generate physically relevant spectra. Indeed, in particle

physics one encounters [12] the measured excitation spectra of the nucleon $(N)$, and the so called $\Delta$ particle, in turn displayed in Fig. $\nabla$

According to an observation due to Refs. 13], 14] and references therein, those spectra repeat with an amazing accuracy the degeneracy patterns of the levels of the electron with spin in the hydrogen atom but are characterized by very different mass splittings. The following mass formula describes pretty well the averaged positions of the three narrow mass bands containing the series of $(n-1)$ parity doubled states (with $n=2$, 4, and 6 ) and with spins ranging from $\frac{1}{2}^{ \pm}$to $\left(n-\frac{3}{2}\right)^{ \pm}$together with the one unpaired maximal spin $\left(n-\frac{1}{2}\right)^{P}$ state of either natural, or, unnatural parity,

$$
M_{(n ; I)}-M_{(n ; I)}^{0}=g_{I} \frac{n^{2}-1}{4}-f_{I} \frac{1}{n^{2}}, \quad I=N, \Delta
$$



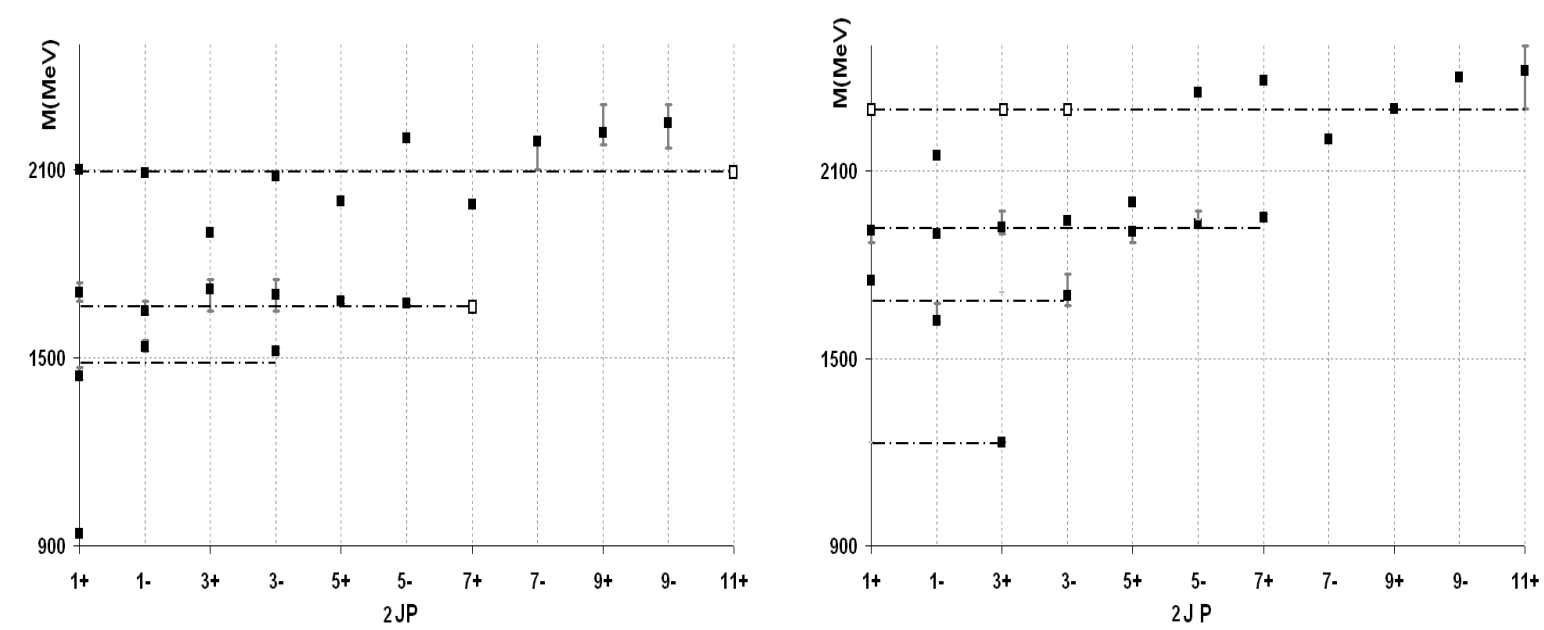

Fig. V. Experimentally observed baryon resonances (l.h.s.) $N$ and (r.h.s.) $\Delta$. The dash-point lines represent the mass average. Notice that the resonances with masses above $2000 \mathrm{MeV}$ are of significantly lower confidence but those with masses below $2000 \mathrm{MeV}$ where the degeneracy is very well pronounced. Empty squares denote predicted ("missing") states.

Comparison of the baryon mass formula with Eq. (47) reveals coincidence between the baryonic and the tRMP spectra. The underlying constituent dynamics of the excited baryons has been found to be that of a quark-di-quark system [13]. In fact, in order to describe the particle spectra one needs to solve the three dimensional Schrödinger equation but this does not cause much problems because upon separation of the variables in polar coordinates the radial part of the three dimensional Schrödinger equation reduces to that very same onedimensional Schrödinger equation (22) up to the centrifugal barrier term, $l(l+1) / z^{2}$ (work in progress). All in all, the trigonometric Rosen-Morse potential and its real orthogonal polynomial solutions open new venues in the calculation of interesting observables in both supersymmetric quantum mechanics and particle spectroscopy.

\section{ACKNOWLEDGMENTS.}

We thank Dr. Alvaro Pérez Raposo for his spontaneous and vivid interest in our results. Work supported by Consejo Nacional de Ciencia y Technología (CONACyT) Mexico under grant number C01-39280. 
[1] E. Witten, Nucl. Phys. B 188, 513 (1981).

[2] C. V. Sukumar, J. Phys. A:Math. Gen. 18, 2917 (1998);

C. V. Sukumar, AIP proceedings 744, eds. R. Bijker et al., Supersymmetries in physics and applications, (New York, 2005), p. 167.

[3] F. Cooper, A. Khare, U. P. Sukhatme, Supersymmetry in Quantum Mechanics, (World Scientific, Singapore, 2001).

[4] R. De, R. Dutt, U. Sukhatme, J. Phys. A: Math. Gen. 25, L843 (1992).

[5] A. B. J. Kuijlaars, A. Martinez-Finkelshtein, R. Orive, Orthogonality of Jacobi Polynomials with General Parameters, E-Print ArXiv: math.CA/0301037 (2003).

[6] B. Beckermann, J. Coussement, W. Van Asshe, E-Print ArXiv: math.CA/0311055 (2003).

[7] Phylippe Dennery, André Krzywicki, Mathematics for Physicists, (Dover, New York, 1996).

[8] M. Abramowitz, I. A. Stegun, Handbook of Mathematical Functions with Formulas, Graphs and Mathematical Tables, (Dover, 2nd edition, New York, 1972).

[9] http://en.wikipedia.org/wiki/Main_Page

[10] C. W. Cryer, Boll. Un. Mat. Ital. 25 (1), 1 (1970);

Renato Álvarez Nodarse, Las funciónes especiales y la fisica matematica (in Spanish), http://euler.us.es/ renato/clases.html

[11] Cliffor Benjamin Compean Jasso, Baryon spectra in a quark-di-quark model with the trigonometric Rosen-Morse potential, (in Spanish), MS thesis, Institute of Physics, Autonomous University of San Luis Potosí, México, September (2005).

[12] L. Alvarez-Gaumé et al., Phys. Lett. B, 592, 1 (2004).

[13] M. Kirchbach, M. Moshinsky, Yu. F. Smirnov, Phys. Rev. D 64, 114005 (2001).

[14] M. Kirchbach, Rev. Mex. Fis. 50, Supl. 2, 54 (2004). 